\title{
A!
}

This is an electronic reprint of the original article.

This reprint may differ from the original in pagination and typographic detail.

Manninen, M.; Nieminen, R.; Hautojärvi, P.; Arponen, J.

\section{Electrons and positrons in metal vacancies}

Published in:

Physical Review B

DOI:

10.1103/PhysRevB.12.4012

Published: $15 / 11 / 1975$

Document Version

Publisher's PDF, also known as Version of record

Please cite the original version:

Manninen, M., Nieminen, R., Hautojärvi, P., \& Arponen, J. (1975). Electrons and positrons in metal vacancies.

Physical Review B, 12(10), 4012-4022. https://doi.org/10.1103/PhysRevB.12.4012

This material is protected by copyright and other intellectual property rights, and duplication or sale of all or part of any of the repository collections is not permitted, except that material may be duplicated by you for your research use or educational purposes in electronic or print form. You must obtain permission for any other use. Electronic or print copies may not be offered, whether for sale or otherwise to anyone who is not an authorised user. 


\title{
Electrons and positrons in metal vacancies
}

\author{
M. Manninen, R. Nieminen, and P. Hautojärvi \\ Department of Technical Physics, Helsinki University of Technology, SF-02150 Espoo 15, Finland
}

\section{J. Arponen}

Research Institute for Theoretical Physics, SF-00170 Helsinki 17, Finland

(Received 14 October 1974; revised manuscript received 16 June 1975)

\begin{abstract}
The electron density profiles at monovacancies of simple metals are calculated by the self-consistent KohnSham method and by a number of statistical methods. The metal is described by a uniform positive background charge together with an interacting electron gas, and the vacancy is approximated as a spherical hole in the background. The Kohn-Sham electron density inside the vacancy is found to be in average $1 / 5$ of the density in the bulk material. Of the various statistical methods, the simple Thomas-Fermi approximation is found to describe best the average electron density over the whole metallic density range when compared to the Kohn-Sham results. The energies of vacancy formation are calculated by using the Kohn-Sham electron densities together with three lattice models, and reasonable numerical success is achieved for alkali metals. In the case of polyvalent metals the results are not satisfactory even if the uniform background were replaced by point ions or if the electron-ion interactions were described by Ashcroft empty-core pseudopotentials. The lifetimes of a positron trapped at the vacancies of several metals are calculated by using both the Kohn-Sham and the Thomas-Fermi electron densities. The results for most metals are in agreement with experimental values. The angular-correlation curve of the positron in aluminum vacancy is calculated directly from the Kohn-Sham one-electron wave functions. The result agrees with the curve calculated from the so-called mixeddensity approximation and also with the experimental result.
\end{abstract}

\section{INTRODUCTION}

The properties of vacancies in metals can be studied in two profoundly different ways. One can assign (usually pairwise) interactions between the atoms using a suitable empirical or semiempirical. description or the interatomic potential of pseudopotential theory. ${ }^{1,2}$ A great advantage of this method is that explicit volume dependence can be eliminated in computing binding energies and thus it can readily be generalized to more complicated defect structures. ${ }^{3}$

A second approach is to start from a given ionic configuration and calculate the conduction-electron distribution around the lattice defect. The relaxed charge density gives rise to a defect potential for electrons and positrons; the former are scattered off it with a concomitant increase in resistivity, while the latter are attracted towards the vacant site and may become trapped. Consequently, the electron density profile is of interest in view of residual resistivity or positron-annihilation parameters. Furthermore, the question of lattice relaxation around a vacancy can be tackled by combining the Kanzaki lattice statics method with the charge distribution calculations for an unrelaxed lattice. ${ }^{4}$

Stott et al. ${ }^{5}$ produced the displaced charge around a lattice vacancy by making the electrons scatter self-consistently in the Hartree sense from a defect potential, for which they chose the negative of the ion field of the missing atom. As the core part of this potential is highly repulsive, their electron densities are, in our opinion, far too low inside the vacancy. Beeby ${ }^{6}$ has developed a Korringa-Kohn-
Rostoker (KKR) type theory for the density of electrons in an imperfect lattice. Its application even to a monovacancy is, however, a fairly lengthy computational task and has not been attempted as yet.

In this paper, we report on calculations of selfconsistent electron density profiles at monovacancies of simple metals, outlined in an earlier paper. ${ }^{7}$ A uniform background-charge model (jellium) together with an interacting electron gas is used. In Sec. II the electron density at the monovacancy is calculated by the density-functional method of Kohn and Sham, ${ }^{8}$ a method which gives a sufficiently accurate description for the jellium model, and in Sec. III the results are compared with those obtained by the Thomas-Fermi method and its various extensions. Using the obtained electron density distributions, we have attempted in Sec. IV to calculate the vacancy -formation energies by a method which parallels the surfaceenergy studies of Lang and Kohn. ${ }^{9}$

The positron-annihilation method has proved useful in studying lattice defects. ${ }^{10}$ The lifetime and angular-correlation measurements yield information about the electron density and momentum distribution around the positron. The disadvantage of the positron-annihilation method is that the positron itself, being a charged particle, distorts the electron distribution. However, the fact that the positron easily gets trapped at defects makes the positron a unique probe in studying the inside $r$ egion of the vacancy. In Sec. $V$ the annihilation characteristics of positrons trapped at vacancies are calculated. The positron lifetime is evaluated for several metals by using both the 
Thomas-Fermi and the Kohn-Sham electron densities. The angular-correlation curve due to trapped positrons is calculated in the case of aluminum, where annihilations with core electrons add only a minor contribution to the curve.

\section{VACANCIES IN THE DENSITY-FUNCTIONAL FORMALISM}

In the uniform-background model a monovacancy is described as a spherical hole in the background; hence the ion density is

$$
n_{+}(\overrightarrow{\mathrm{r}})=n_{0} \Theta\left(r-R_{\mathrm{WS}}\right) \text {. }
$$

Here $n_{0}$ is the background number density, $\theta$ is the step function, and $R_{\mathrm{WS}}$ is the Wigner-Seitz radius satisfying the condition

$$
n_{0} \frac{4}{3} \pi R_{\mathrm{wS}}^{3}=Z \text {, }
$$

where $Z$ is the valence of the metal.

In the formalism of Hohenberg, Kohn, and Sham ${ }^{8,11}$ the total energy of the electronic system is expressed as a functional of the electron density (atomic units are used throughout all formulas),

$$
\begin{aligned}
E[n]= & \int d \overrightarrow{\mathrm{r}} v(\overrightarrow{\mathrm{r}}) n(\overrightarrow{\mathrm{r}})+\frac{1}{2} \iint d \overrightarrow{\mathrm{r}} d \overrightarrow{\mathrm{r}}^{\prime} \frac{n(\overrightarrow{\mathrm{r}}) n\left(\overrightarrow{\mathrm{r}}^{\prime}\right)}{\left|\overrightarrow{\mathrm{r}}-\overrightarrow{\mathrm{r}}^{\prime}\right|} \\
& +T[n]+E_{\mathrm{xc}}[n],
\end{aligned}
$$

where $n(\overrightarrow{\mathrm{r}})$ is the electron number density and $v(\overrightarrow{\mathrm{r}})$ an external potential. $T[n]$ and $E_{\mathbf{x c}}[n]$ are the kinetic and exchange-correlation energies of the system. In the case of a vacancy the external potential $v(\overrightarrow{\mathbf{r}})$ is the electrostatic potential due to the positive background charge $n_{+}$of Eq. (2.1). In the Kohn-Sham method (hereafter referred to as the KS method) one defines an effective one-particle potential

$$
V_{\text {eff }}(\overrightarrow{\mathrm{r}})=-\phi(\overrightarrow{\mathrm{r}})+\delta E_{\mathrm{xc}} / \delta n(\overrightarrow{\mathrm{r}}),
$$

where $\phi(\vec{r})$ is the total electrostatic potential of the system. The electron density is defined as

$$
n(\overrightarrow{\mathrm{r}})=\sum_{i}\left|\psi_{i}(\overrightarrow{\mathrm{r}})\right|^{2},
$$

where the wave functions $\psi_{i}$ are solutions to the Hartree-type one-particle equations

$$
\left[-\frac{1}{2} \nabla^{2}+V_{\text {eff }}(\overrightarrow{\mathrm{r}})\right] \psi_{i}(\overrightarrow{\mathrm{r}})=\epsilon_{i} \psi_{i}(\overrightarrow{\mathrm{r}}) .
$$

The over-all charge balance requires that $\phi(r) \rightarrow 0$ as $r \rightarrow \infty$. Hence the effective potential $V_{\text {eff }}$ approaches the value

$$
\left[\delta E_{\mathrm{xc}} / \delta n(\overrightarrow{\mathrm{r}})\right]_{n=n_{0}} \equiv \mu_{\mathrm{xc}}\left(n_{0}\right) .
$$

The bulk chemical potential is $\mu_{0}=\frac{1}{2} k_{F}^{2}+\mu_{\mathrm{xc}}\left(n_{0}\right)$, $k_{F}$ being the Fermi momentum. The one-particle eigenvalues of Eq. (2.6) are $\epsilon_{k}=\mu_{0}+\frac{1}{2}\left(k^{2}-k_{F}^{2}\right)$, where $k<k_{F}$. Omitting gradient corrections the exchange and correlation part of the effective po- tential can be written

$$
\frac{\delta E_{\mathrm{xc}}}{\delta n(\overrightarrow{\mathrm{r}})}=\frac{d}{d n}\left[n(\overrightarrow{\mathrm{r}}) \epsilon_{\mathrm{xc}}(n(\overrightarrow{\mathrm{r}}))\right],
$$

where $\epsilon_{\mathrm{xc}}$ is the exchange and correlation energy per particle in a homogeneous electron gas of density $n$. The spherical geometry of the problem allows a separation of the angular variables of the wave functions and one is finally led to the following self-consistency problem:

$\left(-\frac{1}{2} \frac{d^{2}}{d r^{2}}+V_{\text {eff }}+\frac{l(l+1)}{2 r^{2}}\right) u_{k, l}=\left[\mu_{0}+\frac{1}{2}\left(k^{2}-k_{F}^{2}\right)\right] u_{k, l}$,

$V_{\text {eff }}(\overrightarrow{\mathrm{r}})=-\phi(\overrightarrow{\mathrm{r}})+\frac{d}{d n}\left[n \epsilon_{\mathrm{xc}}(n)\right]$,

$\nabla^{2} \phi(\overrightarrow{\mathrm{r}})=-4 \pi\left[n_{+}(\overrightarrow{\mathrm{r}})-n(\overrightarrow{\mathrm{r}})\right]$,

$$
n(\overrightarrow{\mathrm{r}})=\frac{1}{\pi^{2}} \int_{0}^{k_{F}} d k k^{2} \sum_{l=0}^{\infty}(2 l+1)\left(\frac{u_{k, l}}{r}\right)^{2} \text {. }
$$

For the correlation energy, we have used the Wigner interpolation formula, so that the exchangecorrelation energy per particle is ${ }^{9}$

$$
\epsilon_{\mathrm{xc}}(n)=-\frac{0.458}{r_{s}(n)}-\frac{0.44}{r_{s}(n)+7.8},
$$

where $r_{s}(n)$ is the conventional density parameter. For comparison, we have also used for the correlation energy a more recent formula of Hedin and Lundqvist, ${ }^{12}$ but the differences in the electron density were found to be less than $1 \%$.

The set of equations (2.8)-(2.11) have been solved numerically by a method described in the Appendix. The results for eight metals, which represent the whole metallic density range, are shown in Fig. 1. The relative electron density inside the vacancy is fairly high. Its dependence on the electron density parameter $r_{s}$ and on the valency $Z$ is shown in Fig. 2 .

In the numerical calculations it was found necessary to use the angular momentum up to $l=7$. In Table I we show the phase shifts at the Fermi energy and the displaced charges corresponding to each partial wave in the case of aluminum. The charge neutrality condition, which equals to the Friedel sum rule, is fulfilled to an accuracy of $1 \%$. The self-consistency achieved in the electron density was better than $0.1 \%$. Outside the vacancy, for $r \gtrsim 3 R_{\mathrm{ws}}$, the electron density reaches its as ymptotic value

$$
n(r)-n_{0} \propto\left[\cos \left(2 k_{F} r+\eta\right)\right] / r^{3},
$$

where $\eta$ is a constant. The first Friedel oscillations in aluminum ard sodium are shown in Fig. 3.

The effective scattering potential $V_{\text {eff }}$ for two representative metals, $\mathrm{Al}$ and $\mathrm{Na}$, are shown in Fig. 4. One can see that in the case of a low-electron density $(\mathrm{Na})$ the exchange-correlation part of 


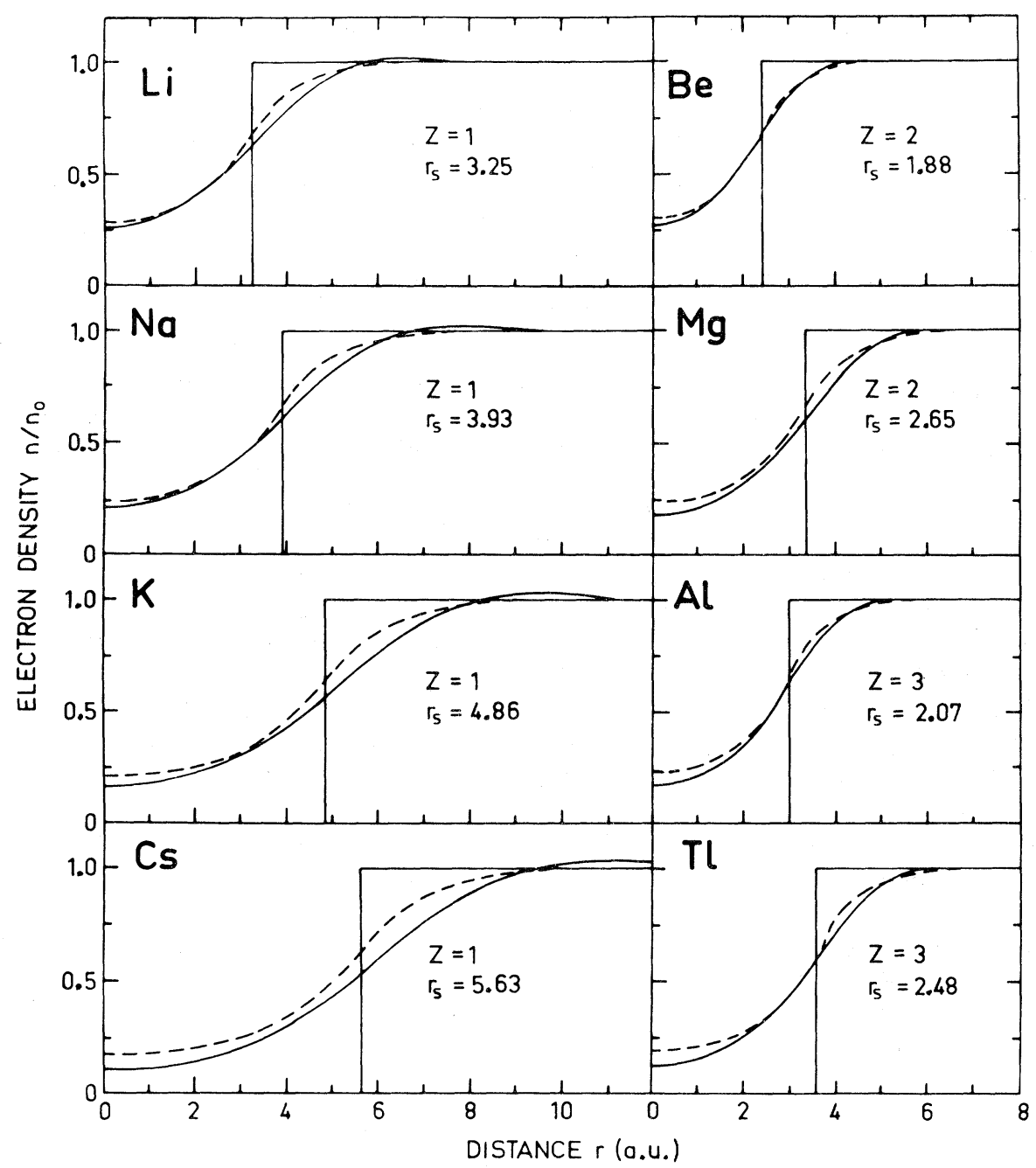

FIG。1. Electron density profiles at vacancies in eight simple metals. The solid lines are the self-consistent KohnSham electron densities, and the dashed ones the Thomas-Fermi results。

the potential becomes dominant. The potentials exhibit also the long-range oscillations similar to those in the electron density.

\section{THOMAS-FERMI METHOD AND ITS EXTENSIONS}

We have also calculated the electron densities at vacancies employing a number of statistical meth-

TABLE I. Scattering phase shifts (in radians) at the Fermi energy and displaced charges corresponding to each angular momentum value.

\begin{tabular}{lcc}
\hline \hline$l$ & $Z_{l}$ & $\delta_{l}\left(k_{F}\right)$ \\
\hline 0 & 0.760 & -1.146 \\
1 & 1.062 & -0.584 \\
2 & 0.732 & -0.228 \\
3 & 0.312 & -0.067 \\
4 & 0.084 & -0.016 \\
5 & 0.026 & -0.004 \\
6 & 0.009 & -0.001 \\
7 & 0.002 & -0.000 \\
\hline
\end{tabular}

ods, which are based on the minimization of the total energy in Eq. (2.3). By taking the kinetic energy in a local description and ignoring the term

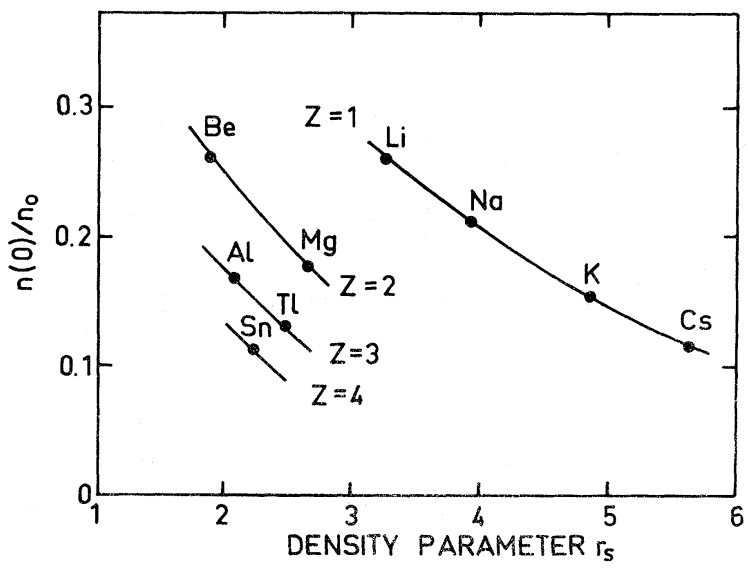

FIG. 2. The relative Kohr im electron density at the centre of the vacancy as a tunction of $r_{s}$ and $Z$. 


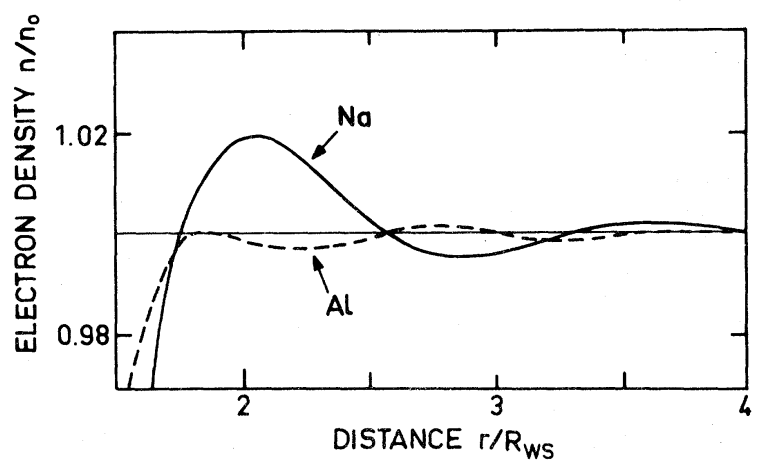

FIG. 3. The first Friedel oscillations in electron densities at vacancies of sodium and aluminum.

$E_{\mathrm{xc}}$, one is led to the Thomas-Fermi (TF) approximation. Including also the local exchange energy in the total energy, one obtains the Thomas-FermiDirac (TFD) approximation. The TF equation in the case of a jellium vacancy was solved by the method described earlier. ${ }^{13}$ The same procedure is used here to calculate the TFD electron densities. The TF electron densities are presented in Fig. 1 as opposed to the KS results. The corresponding electrostatic potentials for aluminum and sodium are shown in Fig. 4.

From atomic calculations ${ }^{14}$ it is well known that the TFD equation is unable to describe the situation where the electron density approaches zero, since the resulting density profile becomes discontinuous. Inside the vacancy the electron densities for all metals are so low that this happens, and consequently the TFD method seems to be quite useless for vacancy calculations.

The inhomogeneity corrections to the total energy can be taken into account by density gradients using the method of Hohenberg and Kohn. ${ }^{11}$ The first correction to the kinetic energy is of the Weizsäcker type

$$
E_{W}=\frac{1}{72} \int d \overrightarrow{\mathbf{r}} \frac{|\nabla n|^{2}}{n}
$$

first suggested by Kirzhnits. ${ }^{15}$ The original Weizsäcker term ${ }^{16}$ had the coefficient $\frac{1}{8}$ instead of the coefficient $\frac{1}{72}$. Jones and Young ${ }^{17}$ have proposed that when the variations in electron density are rapid one should rather use the original coefficient $\frac{1}{8}$. In the case of a vacancy, however, the use of the larger factor leads to much higher interior densities than those of the KS method. Including Eq. (3.1) into Eq. (2.3), one arrives at the nonlinear integrodifferential equation [which we shall call the Thomas-Fermi-Dirac-Weizsäcker (TFDW) equation]

$\frac{1}{2}\left(3 \pi^{2}\right)^{3 / 2} n^{3 / 2}-(3 / \pi)^{1 / 3} n^{1 / 3}+\frac{1}{72} \frac{(\nabla n)^{2}}{n^{2}}-\frac{1}{36} \frac{\nabla^{2} n}{n}$

$$
-\int d \overrightarrow{\mathbf{r}}^{\prime} \frac{n_{+}\left(\overrightarrow{\mathbf{r}}^{\prime}\right)-n(\overrightarrow{\mathbf{r}})}{\left|\overrightarrow{\mathbf{r}}-\overrightarrow{\mathbf{r}}^{\prime}\right|}=\mu
$$

where $\mu$ is the chemical potential.

In the Hohenberg-Kohn formalism, ${ }^{11}$ one can generate gradient corrections for the exchange energy also. The first such correction is of the form $-\gamma \int d \overrightarrow{\mathbf{r}}|\nabla n|^{2} / n^{4 / 3}$, where the positive factor $\gamma$ depends strongly on the dielectric function used to calculate the gradient expansion, as shown by Geldart et al. ${ }^{18}$ Because of the negative sign of this correction term, the energy functional (2.3) is no longer bounded from below and so it cannot be used in calculating the electron density by a minimization principle. Recently, Niklasson et al. ${ }^{19}$ have calculated a gradient correction to the correlation energy. It is also of the form $\int d \overrightarrow{\mathbf{r}}|\nabla n|^{2} / n^{4 / 3}$, but positive and so large that, combined with the exchange term, it makes a gradient correction, which is positive and thus reasonable for use in the functional (2.3).

From Fig. 1 one can see that the TF result describes fairly well the average electron density in the vacancy over the whole metallic density range $\left(1.8 \leqslant r_{s} \leqslant 5.6\right)$. Figure 5 shows the differences of the TF and TFDW electron densities from the KS

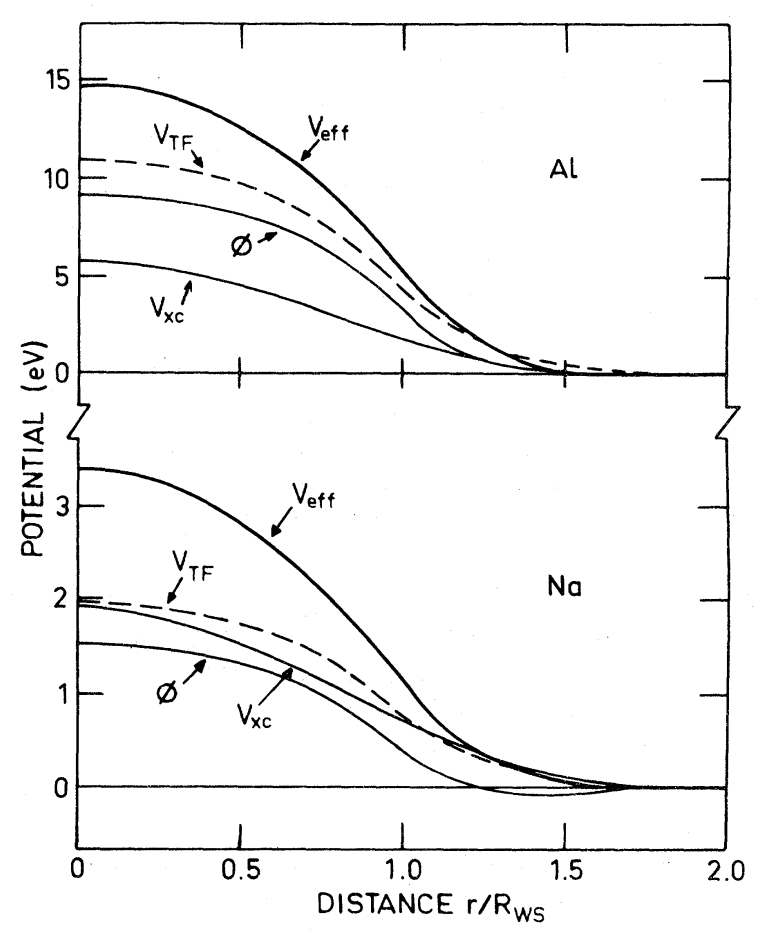

FIG. 4. Effective scattering potentials for electrons in the vacancies of sodium and aluminum. $V_{\text {eff }}$ is the total scattering potential in the Kohn-Sham model, $\phi$ is the electrostatic and $V_{\mathrm{xc}}$ the exchange-correlation part of this potential. $V_{\mathrm{TF}}$ is the electrostatic potential in the Thomas-Fermi approximation. 


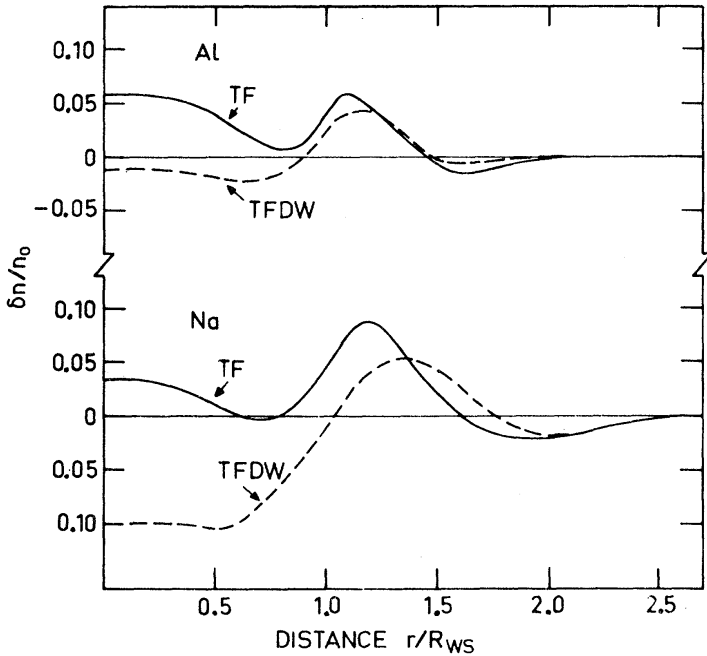

FIG. 5. Deviations of the Thomas-Fermi (TF) and Thomas-Fermi-Dirac-Weizsäcker (TFDW) electron densities at vacancies from the Kohn-Sham result in aluminum and sodium.

density in the cases of $\mathrm{Al}$ and $\mathrm{Na}$. The TFDW result is slightly better than the TF result in a highelectron-density metal like $\mathrm{Al}$, but worse in the case of the low electron density of $\mathrm{Na}$, since the Dirac term overestimates the exchange effect at low electron densities. The statistical methods, with or without gradient corrections, cannot yield the correct Friedel oscillations which are present in the $\mathrm{KS}$ electron densities.

It is also interesting to compare the TF and TFW (TFDW without exchange) densities with the Hartree electron density, because none of these methods takes into account any exchange or correlation phenomena. The Hartree density is obtained by including only the electrostatic part into the effecttive potential $V_{\text {ef } f}$ in Eq. (2.8). Ir respective of the bulk $r_{s}$ the Hartree electron density inside the vacancy becomes slightly greater than the TF density. The gradient correction (3.1) brings the TF result closer to the Hartree density, and its effect is larger in high-density metals. It can also be noted that the amplitudes of the Friedel oscillations produced by the Hartree calculations are for low-density metals about one order of magnitude smaller than the amplitudes in the $\mathrm{KS}$ electron densities.

In the computations described above the background charge was taken to be a step function, Eq. (2.1). In order to test if the discontinuity in the background charge has any effect on the electron density, we also calculated the TF densities by using a smeared-out background charge distribution. It was found that the electron densities were rather insensitive to the exact shape of the background charge, if the smearing was constrained within reasonable limits.

\section{VACANCY-FORMATION ENERGY}

In this section we use the electron densities discussed above to calculate the formation energies of vacancies in simple metals. Our approach is analogous to that taken by Lang and $\mathrm{Kohn}^{9}$ in their study of energetics of metal surfaces. For reference to other methods we refer to a recent article of Minchin et al. ${ }^{20}$

We ignore lattice relaxation just as Lang and Kohn did, although relaxation certainly is more important for the vacancy-formation energy than for the electron density distribution. The reason is that the vacancy-formation energy is a sum of large terms which almost exactly cancel each other; thus even a small effect due to relaxation may have a relatively large contribution to the small vacancy-formation energy. ${ }^{21}$ The magnitude of the relaxation of the nearest atoms, e.g., in $\mathrm{Al}$, is about $(2-3) \%$ of the lattice constant. ${ }^{22}$

Within the density functional formalism the vacancy-formation energy can be expressed as a sum of three terms

$$
E_{v}=\Delta T+\Delta E_{\mathrm{xc}}+\Delta E_{c}
$$

which are the differences in kinetic, exchange-correlation, and electrostatic energies between a perfect lattice of $N$ atoms and a lattice with one atomic cell removed from the bulk and placed on the surface. The change in the kinetic energy is

$\Delta T=\sum_{k} \Delta \epsilon_{k}-\frac{2}{5} Z E_{F}+N Z \mu_{\mathrm{xc}}\left(n_{0}\right)-\int_{\Omega} d \overrightarrow{\mathrm{r}} V_{\mathrm{eff}}(\overrightarrow{\mathrm{r}}) n(\overrightarrow{\mathrm{r}})$.

The first term in Eq. (4.2) is because of the shifts of the one-electron eigenvalues, the second arises from the displacement of the Fermi energy as the total volume of the system is increased. In the final term $\Omega$ is the total volume of the final $N+1$ unit cells. The corresponding change in the total exchange-correlation energy is

$$
\Delta E_{\mathrm{xc}}=\int_{\Omega} d \overrightarrow{\mathrm{r}} \epsilon_{\mathrm{xc}}(n) n(\overrightarrow{\mathrm{r}})-N Z \epsilon_{\mathrm{xc}}\left(n_{0}\right) .
$$

The sum of the eigenvalue deviations can be written in terms of the phase shifts of the scattered electrons, ${ }^{5}$ whence we get for the vacancy-formation energy

$$
\begin{aligned}
E_{v}= & -\frac{2}{\pi} \sum_{l=0}^{\infty}(2 l+1) \int_{0}^{k_{F}} d k k \delta_{l}(k)-\frac{2}{5} Z E_{F}+\int_{\Omega} d \overrightarrow{\mathrm{r}} \phi(\overrightarrow{\mathrm{r}}) n(\overrightarrow{\mathrm{r}}) \\
& +Z\left[\epsilon_{\mathrm{xc}}\left(n_{0}\right)-\mu_{\mathrm{xc}}\left(n_{0}\right)\right]+\int_{\Omega} d \overrightarrow{\mathrm{r}}\left[\epsilon_{\mathrm{xc}}(n) n(\overrightarrow{\mathrm{r}})-\epsilon_{\mathrm{xc}}\left(n_{0}\right) n_{0}\right. \\
& \left.-\mu_{\mathrm{xc}}(n) n(\overrightarrow{\mathrm{r}})+\mu_{\mathrm{xc}}\left(n_{0}\right) n_{0}\right]+\Delta E_{c} .
\end{aligned}
$$

Using different descriptions of the ionic lattice, we 
TABLE II. Vacancy-formation energies in eV for different lattice models calculated from the Kohn-Sham electron densities. The numerical uncertainty is about $2 \%$.

\begin{tabular}{lccccccc}
\hline \hline Metal & Lattice & $Z$ & $r_{s}$ & Jellium & Point ion & Pseudo ion & Experimental \\
\hline $\mathrm{Li}$ & bcc & 1 & 3.25 & 0.26 & 0.83 & 0.25 & $0.34^{\mathrm{a}}$ \\
$\mathrm{Na}$ & bcc & 1 & 3.93 & 0.18 & 0.46 & 0.38 & $0.42^{\mathrm{b}}$ \\
$\mathrm{K}$ & bcc & 1 & 4.86 & 0.17 & 0.19 & 0.34 & $0.39^{\mathrm{e}}$ \\
$\mathrm{Cs}$ & bcc & 1 & 5.63 & 0.18 & 0.07 & 0.35 & $0.28^{\mathrm{d}}$ \\
$\mathrm{Be}$ & hcp & 2 & 1.88 & -2.17 & 1.28 & -1.38 & \\
$\mathrm{Mg}$ & hcp & 2 & 2.65 & -0.22 & 0.93 & 0.76 & $0.81^{\mathrm{e}}$ \\
$\mathrm{Al}$ & fcc & 3 & 2.07 & -1.20 & 2.94 & 1.56 & $0.66^{\mathrm{f}}$ \\
$\mathrm{Tl}$ & hcp & 3 & 2.48 & -0.05 & 1.89 & & \\
\hline \hline
\end{tabular}

${ }^{2} \mathrm{R}$. Feder, Phys. Rev. B 2, 828 (1970).

${ }^{\mathrm{b}} \mathrm{R}$. Feder and H. P. Charbnau, Phys. Rev. 149, 464 (1966).

${ }^{C}$ D. K. MacDonald, J. Chem. Phys. 21, 177 (1953).

${ }^{d}$ Ya. A. Kraftmacher and P. G. Strelkov, in Vacancies and Interstitials in Metals, edited by A. Seeger, D. Schumacher, W. Schilling, and J. Diehl (NorthHolland, Amsterdam, 1970), p. 59.

${ }^{\circ} \mathrm{C}$. Mairy, J. Hillairet, and D. Schumacher, Acta Metall. 15, 1258 (1967).

${ }^{{ }^{\prime}}$ B. T. A. McKee, W. Triftshäuser, and A. T. Stewart, Phys. Rev. Lett. $\underline{28}, 358$ (1972).

now proceed to evaluate the electrostatic contribution $\Delta E_{c}$ for insertion in Eq. (4.4) to obtain the vacancy-formation energy. The results are reported in Table II.

\section{A. Jellium model}

In the uniform-background model the change in the Coulomb energy $E_{c}$ is simply

$$
\Delta E_{c}=\frac{1}{2} \int_{\Omega} d \overrightarrow{\mathbf{r}} \phi(\overrightarrow{\mathrm{r}})\left[n_{+}(\overrightarrow{\mathrm{r}})-n(\overrightarrow{\mathrm{r}})\right],
$$

where $\phi$ is the electrostatic potential. From the appropriate column in Table II it is seen that, whereas the simple jellium approximation is fairly adequate for the monovalent and low-density alkalis, it breaks down for larger $Z$ and higher electron density. A similar trend is clear in the Lang and $\mathrm{Kohn}^{9}$ results for surface energies.

\section{B. Point-ion model}

We believe that the use of the jellium model causes its largest error in the electrostatic energy, whereas the electron density profile should be quite adequate in the average for simple metals. Therefore one can go beyond the jellium model by perturbatively evaluating the corrections due to the discreteness of the ionic lattice. If one simply describes the ion cores by point charges $Z$, the total electrostatic energy $E_{c}$ can be written as

$$
E_{c}=E_{\mathrm{ii}}+E_{\mathrm{ie}}+E_{\theta \theta}
$$

where

$$
E_{\mathrm{i} i \mathrm{i}}=\frac{Z^{2}}{2} \sum_{j \neq j} \frac{1}{\left|\overrightarrow{\mathrm{R}}_{i}-\overrightarrow{\mathrm{R}}_{j}\right|},
$$

$$
\begin{aligned}
& E_{\mathrm{ie}}=-Z \sum_{i} \int d \overrightarrow{\mathrm{r}} \frac{n(\overrightarrow{\mathrm{r}})}{\left|\overrightarrow{\mathrm{r}}-\overrightarrow{\mathrm{R}}_{i}\right|}, \\
& E_{\mathrm{e \theta}}=\frac{1}{2} \iint d \overrightarrow{\mathrm{r}} d \overrightarrow{\mathrm{r}}^{\prime} \frac{n(\overrightarrow{\mathrm{r}}) n\left(\overrightarrow{\mathrm{r}}^{\prime}\right)}{\left|\overrightarrow{\mathrm{r}}-\overrightarrow{\mathrm{r}}^{\prime}\right|} .
\end{aligned}
$$

These terms represent ion-ion, electron-ion, and electron-electron interactions. $\vec{R}_{i}$ are the position vectors of the ions. We denote by $U_{0}(\overrightarrow{\mathrm{r}})$ the total electrostatic potential in the unperturbed point-ion-uniform-electron system and define a constant $U_{00}$,

$U_{00}=\lim _{\overrightarrow{\mathbf{r}} \rightarrow 0}\left(U_{0}(\overrightarrow{\mathrm{r}})-\frac{Z}{r}\right)=\sum_{i} \frac{Z}{\left|\overrightarrow{\mathrm{R}}_{i}\right|}-\int d \overrightarrow{\mathbf{r}} \frac{n_{0}}{|\overrightarrow{\mathbf{r}}|}$.

As one unit cell is removed from the lattice the energies (4.7)-(4.9) change by the amounts (taking the vacant site as the origin),

$$
\begin{aligned}
\Delta E_{1 \mathrm{i}}= & -Z U_{00}-Z \int d \overrightarrow{\mathrm{r}} \frac{n_{0}}{|\overrightarrow{\mathrm{r}}|}, \\
\Delta E_{\mathrm{i} \theta}= & -\int d \overrightarrow{\mathrm{r}}\left[n(\overrightarrow{\mathrm{r}})-n_{0}\right] U_{0}(\overrightarrow{\mathrm{r}}) \\
& +\iint d \overrightarrow{\mathrm{r}} d \overrightarrow{\mathrm{r}}^{\prime} \frac{\left[n(\overrightarrow{\mathrm{r}})-n_{0}\right] n_{0}}{\left|\overrightarrow{\mathrm{r}}-\overrightarrow{\mathrm{r}}^{\prime}\right|}-Z \int d \overrightarrow{\mathrm{r}} \frac{n(\overrightarrow{\mathrm{r}})}{|\overrightarrow{\mathrm{r}}|}, \\
\Delta E_{0 \theta}= & \frac{1}{2} \iint d \overrightarrow{\mathrm{r}} d \overrightarrow{\mathrm{r}}^{\prime} \frac{n(\overrightarrow{\mathrm{r}}) n\left(\overrightarrow{\mathrm{r}}^{\prime}\right)-n_{0}^{2}}{\left|\overrightarrow{\mathrm{r}}-\overrightarrow{\mathrm{r}}^{\prime}\right|} .
\end{aligned}
$$

The electrostatic self-energy of the removed unit cell can be written in the form

$$
E_{c, \text { unit cell }}=\frac{Z}{2} U_{00}-\frac{1}{2} \int d \overrightarrow{\mathrm{r}} n_{0} U_{0}(\overrightarrow{\mathrm{r}}) \text {. }
$$


Combining Eqs. (4.11)-(4.14) gives the total electrostatic contribution

$$
\begin{aligned}
\Delta E_{c}= & -\frac{Z}{2} U_{00}-\frac{1}{2} \int d \overrightarrow{\mathrm{r}} n_{0} U_{0}(\overrightarrow{\mathrm{r}})-\int d \overrightarrow{\mathrm{r}}\left[n(\overrightarrow{\mathrm{r}})-n_{0}\right] U_{0}(\overrightarrow{\mathrm{r}}) \\
& +\frac{1}{2} \iint d \overrightarrow{\mathrm{r}} d \overrightarrow{\mathrm{r}}^{\prime} \frac{\left[n(\overrightarrow{\mathrm{r}})-n_{0}\right]\left[n\left(\overrightarrow{\mathrm{r}}^{\prime}\right)-n_{0}\right]}{\left|\overrightarrow{\mathrm{r}}-\overrightarrow{\mathrm{r}}^{\prime}\right|} \\
& +Z \int d \overrightarrow{\mathrm{r}} \frac{n(\overrightarrow{\mathrm{r}})-n_{0}}{|\overrightarrow{\mathrm{r}}|}
\end{aligned}
$$

In this formula every term can easily be computed numerically. When calculating the lattice sums inherent in the potential function of the perfect crystal, $U_{0}(\overrightarrow{\mathrm{r}})$, we have used up to 15 th-nearestneighbor shells. The vacancy-formation energies with $\Delta E_{c}$ from Eq. (4.15) (Table II) are positive for all the metals considered, but rather large. In the case of alkalis, the point-ion model is generally a setback from the jellium approximation.

$$
\text { C. Pseudo-ion model }
$$

An obvious improvement of the crude point-ion model is to replace the pure Coulomb potential by an electron-ion pseudopotential. We have chosen to use the Ashcroft empty-core potential ${ }^{23}$

$$
V_{\mathrm{ps}}(\overrightarrow{\mathrm{r}})=\left\{\begin{array}{cc}
0, & r<r_{c} \\
-Z / r, & r>r_{c}
\end{array} .\right.
$$

For the cutoff radius $r_{c}$ we have used the values of Shuy and Gaspari, ${ }^{24}$ which are, in fact, very near to the original values of Ashcroft. ${ }^{23}$ Defining the potential function in the pseudo-ion lattice as

$$
W_{0}(\overrightarrow{\mathrm{r}})=Z \sum_{i} \frac{\Theta\left(\left|\overrightarrow{\mathrm{R}}_{i}-\overrightarrow{\mathrm{r}}\right|-r_{c}\right)}{\left|\overrightarrow{\mathrm{r}}-\overrightarrow{\mathrm{R}}_{i}\right|}-\int d \overrightarrow{\mathrm{r}}^{\prime} \frac{n_{0}}{\left|\overrightarrow{\mathrm{r}}-\overrightarrow{\mathrm{r}}^{\prime}\right|},
$$

we end up with a result analogous to Eq. (4.15).

$$
\begin{aligned}
\Delta E_{c}= & -\frac{Z}{2} U_{00}-\int d \overrightarrow{\mathrm{r}} n_{0} W_{0}(\overrightarrow{\mathrm{r}})+\frac{1}{2} \int d \overrightarrow{\mathrm{r}} n_{0} U_{0}(\overrightarrow{\mathrm{r}})-\int d \overrightarrow{\mathrm{r}}\left[n(\overrightarrow{\mathrm{r}})-n_{0}\right] W_{0}(\overrightarrow{\mathrm{r}}) \\
& +\frac{1}{2} \iint d \overrightarrow{\mathrm{r}} d \overrightarrow{\mathrm{r}}^{\prime} \frac{\left[n(\overrightarrow{\mathrm{r}})-n_{0}\right]\left[n\left(\overrightarrow{\mathrm{r}}^{\prime}\right)-n_{0}\right]}{\left|\overrightarrow{\mathrm{r}}-\overrightarrow{\mathrm{r}}^{\prime}\right|}+Z \int d \overrightarrow{\mathrm{r}} \frac{n(\overrightarrow{\mathrm{r}}) \theta\left(r-r_{c}\right)-n_{0}}{|\overrightarrow{\mathrm{r}}|} .
\end{aligned}
$$

The computed vacancy-formation energies, again in Table II, are closer to experimental values than those of the point-ion model, but, apart from the alkali metals, not very satisfactory. The discrepancies are larger for higher-valent metals, and for Be even a negative value is obtained.

The pseudopotential theory together with the electron density calculated from the uniform-background model does not seem to yield consistently accurate vacancy-formation energies, as opposed to the surface energies, which, by a similar method, are in fairly good agreement with experimental results. ${ }^{9}$ Part of the disagreement here can be explained by lattice relaxation around the vacancy, which would tend to lower the energy values.

The substantial discrepancies for higher valence indicate the breakdown of a perturbative evaluation of the electrostatic terms; in a more successful formulation, the self-consistent screening of the ionic charges would have to be incorporated. Alternatively, one can generate corrections to the jellium problem by screening linearly the difference potential between jellium and discrete ions, a scheme devised by Finnis. ${ }^{25}$ An advantage of the present method is that the vacancy-formation energies are not extremely sensitive to variations in the pseudo-potential parameters, which is often the case in pair (or pseudo) potential formulations. ${ }^{26}$

\section{POSITRON ANNIHILATION AT METAL VACANCIES}

Factors contributing to the positron trapping in defects of metals have been described in detail in Ref. 13. The trapping potential consists of three ingredients,

$$
V=-E_{0} \Theta\left(r-R_{\mathrm{wS}}\right)+V_{\text {corr }}+\phi
$$

The first term on the right-hand side is due to the lack of a positive ion at the vacancy; the reduction of the kinetic energy of the positron is accounted for by a square well with a depth $E_{0}$ equal to the positron zero-point energy in a perfect lattice. ${ }^{27}$ $V_{\text {corr }}$ is the change in the electron-positron correlation energy taken to be dependent on the local electron density. We have used the numerical values of Bhattacharyya and Singwi. ${ }^{28} \phi$ is the electrostatic potential of the vacancy defined in Sec. II.

The ground-state wave function of the positron at the vacancy has been calculated numerically by using both $\mathrm{KS}$ and $\mathrm{TF}$ electron densities. The positron-vacancy binding energies in various metals are shown in Table III. The smaller binding energies in the KS model are mainly due to the smallex 
TABLE III. The binding energies of positrons in vacancies in the Kohn-Sham (KS) and Thomas-Fermi (TF) models. $P$ is the probability of the trapped positron to be outside the vacancy.

\begin{tabular}{lccrr}
\hline & \multicolumn{2}{c}{$E_{b}(\mathrm{eV})$} & \multicolumn{2}{c}{$P(\%)$} \\
Metal & $\mathrm{KS}$ & $\mathrm{TF}$ & \multicolumn{1}{c}{ KS } & $\mathrm{TF}$ \\
\hline $\mathrm{Li}$ & 0.00 & 0.04 & 100 & 85 \\
$\mathrm{Na}$ & 0.02 & 0.19 & 85 & 66 \\
$\mathrm{~K}$ & 0.02 & 0.26 & 80 & 55 \\
$\mathrm{Cs}$ & 0.01 & 0.26 & 82 & 51 \\
$\mathrm{Be}$ & 0.80 & 1.32 & 59 & 54 \\
$\mathrm{Mg}$ & 0.59 & 1.09 & 51 & 45 \\
$\mathrm{Al}$ & 1.75 & 2.60 & 38 & 33 \\
$\mathrm{Tl}$ & 1.72 & 2.50 & 32 & 28 \\
$\mathrm{Sn}$ & 2.64 & 3.59 & 25 & 23 \\
\hline \hline
\end{tabular}

electrostatic potential $\phi$ in this model. In the case of lithium there is no bound state in the KS model and the binding is very weak also for other alkali metals. Table III also shows the probabilities $P$ of the trapped positron to stay outside of the vacancy. This probability is defined as

$$
P=4 \pi \int_{R_{\mathrm{WS}}}^{\infty} d r r^{2}\left|\psi_{+}(r)\right|^{2}
$$

where $\psi_{+}$is the ground-state wave function of the trapped positron. As is natural, the smaller binding energies in the $\mathrm{KS}$ model produce slightly higher values of $P$.

The annihilation rate of the positron in an inhomogeneous electron gas can be approximated by ${ }^{13,29}$

$$
\lambda=\int d \overrightarrow{\mathrm{r}}\left|\psi_{+}(\overrightarrow{\mathrm{r}})\right|^{2} \Gamma(n(\overrightarrow{\mathrm{r}})),
$$

where $\Gamma(n)$ is the annihilation rate for a homogeneous electron gas. For $\Gamma$ we use the expression suggested by Brandt and Reinheimer ${ }^{29}$

$$
\begin{aligned}
\Gamma(n) & =(2+134 n) \times 10^{9} \mathrm{sec}^{-1} \\
& =\frac{12}{r_{s}^{3}}\left(1+\frac{10+r_{s}^{3}}{6}\right) \times 10^{9} \mathrm{sec}^{-1} .
\end{aligned}
$$

The effect of annihilations with core electrons can be roughly taken into account in a way proposed by West. ${ }^{30}$ The annihilation rate in a perfect solid is calculated from Eq. (5.4) by using a renormalized electron density

$$
n^{\prime}=n_{0}\left(1+\Gamma_{c} / \Gamma_{v}\right)
$$

where $\Gamma_{c}$ and $\Gamma_{v}$ are the annihilation rates with core and valence electrons, respectively. The ratio $\Gamma_{c} / \Gamma_{v}$ can be estimated from angular-correlation measurement. Following West ${ }^{30}$ we take $\Gamma_{c} /\left(\Gamma_{v}\right.$ $\left.+\Gamma_{c}\right)=0.80 A_{c} /\left(A_{c}+A_{v}\right)$, where $A_{c}$ and $A_{v}$ are the areas of core and valence electron parts of the angular-correlation curve. The positron can annihilate with core electrons only outside the vacancy, so that the electron density used in Eq. (5.3) to calculate the total annihilation rate for a trapped positron is

$$
n^{\prime}(\overrightarrow{\mathrm{r}})=\left\{\begin{array}{cc}
n(\overrightarrow{\mathrm{r}}), & r<R_{\mathrm{ws}} \\
n(\overrightarrow{\mathrm{r}})+\Gamma_{c} n_{0} / \Gamma_{v}, & r>R_{\mathrm{ws}}
\end{array} .\right.
$$

Here $n(\overrightarrow{\mathbf{r}})$ is the electron density in the vacancy, calculated in Secs. II and III.

The results for positron lifetimes for several metals are shown in Table IV. In such cases where the KS electron densities are not calculated, we give the lifetimes obtained from the TF elec-

TABLE IV. Positron lifetimes in psec at metal va-

\begin{tabular}{|c|c|c|c|c|c|}
\hline Metal & $\begin{array}{r}A_{c} / A \\
(\%) \\
\end{array}$ & theory & $\begin{array}{l}\tau_{b} \\
\text { experiment }\end{array}$ & theory & $\begin{array}{l}\tau_{v} \\
\text { experiment }\end{array}$ \\
\hline $\mathrm{Li}$ & $17^{\mathrm{a}}$ & 325 & $291^{b}$ & $325^{*}$ & $* *$ \\
\hline $\mathrm{Na}$ & $19^{\mathrm{a}}$ & 381 & $338^{\mathrm{b}}$ & $391^{*}$ & ** \\
\hline $\mathrm{K}$ & $25^{\mathrm{c}}$ & 426 & $397^{b}$ & $436^{*}$ & ** \\
\hline $\mathrm{Rb}$ & $28^{\circ}$ & 436 & $406^{b}$ & 458 & $* *$ \\
\hline $\mathrm{Cs}$ & $35^{\mathrm{c}}$ & 445 & $418^{\mathrm{b}}$ & $452^{*}$ & $* *$ \\
\hline $\mathrm{Be}$ & $6^{\mathrm{a}}$ & 142 & $213^{\mathrm{b}}$ & $177^{*}$ & \\
\hline $\mathrm{Mg}$ & $18^{\mathrm{d}}$ & 249 & $235^{\circ}$ & $307^{*}$ & $255^{\theta}$ \\
\hline $\mathrm{Zn}$ & $49^{d}$ & 161 & $179^{t}$ & 233 & $240^{t}$ \\
\hline $\mathrm{Cd}$ & $57^{d}$ & 185 & $196^{t}$ & 276 & $232^{f}$ \\
\hline $\mathrm{Hg}$ & $71^{\mathrm{B}}$ & 169 & $138^{\mathrm{h}}$ & 266 & $165^{\mathrm{h}}$ \\
\hline $\mathrm{Al}$ & $17^{d}$ & 162 & $161^{i}$ & $237^{*}$ & $243^{1}$ \\
\hline $\mathrm{Ga}$ & $39^{\mathrm{B}}$ & 156 & $190^{f}$ & 248 & $260^{J}$ \\
\hline In & $41^{d}$ & 185 & $182^{k}$ & 289 & $240^{k}$ \\
\hline $\mathrm{Tl}$ & $38^{8}$ & 199 & $210^{\theta}$ & $302^{*}$ & $230^{\circ}$ \\
\hline Sn & $32^{d}$ & 167 & $202^{b}$ & $282^{*}$ & \\
\hline $\mathrm{Pb}$ & $42^{d}$ & 168 & $220^{\circ}$ & 291 & $274^{e}$ \\
\hline $\mathrm{Cu}$ & $73^{d}$ & 163 & $132^{1}$ & 205 & \\
\hline $\mathrm{Ag}$ & $89^{d}$ & 164 & & 227 & \\
\hline $\mathrm{Au}$ & $96^{\mathrm{d}}$ & 143 & $121^{1}$ & 212 & $211^{i}$ \\
\hline
\end{tabular}
cancies. $\tau_{b}$ is the bulk lifetime and $\tau_{v}$ is the lifetime of the trapped positron. $A_{c} / A$ is the core contribution in the angular-correlation curve. *Calculated from the Kohn-Sham electron density. **Experiments seem to show that no trapping occurs in these metals (Ref. 31).

${ }^{8} \mathrm{G}$. Lang and S. De Benedetti, Phys. Rev。 108, 914 (1957).

${ }^{b}$ H. Weisberg and S. Berko, Phys. Rev. 154, 249 (1967)。

${ }^{c}$ Estimated from the curves of Stewart, Can. J. Phys. 35, 168 (1957).

${ }^{\mathrm{d}}$ T. E. Jackman, M. Sc. thesis (University of Guel.ph, 1974) (unpublished).

${ }^{\circ}$ B. T. A. McKee (private communication).

${ }^{\text {II }}$. K. MacKenzie, T. L. Khoo, A. B. McDonald, and B. T. A. McKee, Phys. Rev. Lett. 19, 946 (1967).

8. H. Kusmiss, Ph. D. thesis (University of North Carolina, 1965) (unpublished).

'J. D. McGervey, in Positron Annihilation, edited by A. J. Stewart and L. Roellig (Academic, New York, 1967), p. 305 .

'T. M. Hall, A. N. Goland, and C. L. Snead, Jr., Phys. Rev. B 10, 3062 (1974).

${ }^{j}$ W. Brandt and H. F. Waung, Phys, Lett. 27A, 700 (1968)。

${ }^{\mathrm{k}} \mathrm{V}$. H. C。Crisp, D. G. Lock, and R. N. West, J. Phys. F $\underline{4}, 830$ (1974).

${ }^{1}$ P. Hautojärvi and P. Jauho, Acta Polytech. Scand. Phys. Incl. Nucleon Ser. 98, 1 (1973). 
tron densities. The differences in the lifetimes calculated by using either the TF or the KS electron densities were found to be between 0 and $3 \%$, and thus insignificant compared to the uncertainties in the experimental values. The positron lifetimes both in the bulk and when captured at a vacancy agree in most metals fairly well with the experimental results. Since the core annihilation was approximated in a very crude manner, it is natural that the results are better for simple metals where the core contribution is small. In the case of alkali metals there is no experimental evidence of positron trapping at vacancies. ${ }^{31}$ For these metals our calculations give such small binding energies that the trapping does not seem probable. Even if it occurs, the lifetime of the trapped positron differs so little from the bulk value that trapping is difficult to observe experimentally.

Recently, Brandt ${ }^{32}$ has calculated the lifetimes of positrons trapped at metal vacancies by a different approach. In his model the electron density is calculated in a TF approximation, but including also the trapped positron as a stationary external positive-charge distribution contributing to the self-consistent electrostatic potential. On the other hand, the electron density found in this way together with the positive background contributes to the electrostatic potential, which is used as the trapping potential of the positron. The binding energies of the positrons are, because of the strong attractive potential, much bigger than in our results, e.g., in Al the binding energy is $6.0 \mathrm{eV}$. We believe that this method does not take into account correctly the screening of the positron in the electron system, since it totally ignores the fact that the positron is already screened in the perfect lattice, and consequently the theory leads to an electrostatic potential too deep inside the vacancy. The dimensions of the vacancy are large in comparison to the screening cloud around the positron, and therefore, qualitatively speaking, the positron and its screening cloud follow each other and move together inside the vacancy, which situation is contradictory to the one assumed in the model of Ref. 32.

The second quantity to be acquired from annihilation experiments is the angular dependence of the two annihilation quanta, which is related to the momentum distribution of the electron-positron system. In the independent-particle model the momentum density of the annihilating electron-positron pair is ${ }^{10}$

$$
\rho(\overrightarrow{\mathrm{p}}) \propto \sum_{i}\left|\int d \overrightarrow{\mathrm{r}} e^{-i \overrightarrow{\mathrm{p}} \cdot \overrightarrow{\mathrm{r}}} \psi_{+}(\overrightarrow{\mathrm{r}}) \psi_{i}(\overrightarrow{\mathrm{r}})\right|^{2} .
$$

The angular-correlation curve, i.e., the momentum distribution of the $p_{z}$ component, is obtained by integrating $\rho(\overrightarrow{\mathrm{p}})$ over $p_{x}$ and $p_{y}$, and for an iso- tropic distribution one can write

$$
I\left(p_{z}\right)=\int_{p_{z}}^{\infty} d p p \rho(p) .
$$

The one-particle functions in the KS model are not, in principle, the proper electron wave functions, and it may be questioned whether Eq. (5.7) reflects the true momenta in the interacting system. The equivalent problem in the Compton profiles of inhomogeneous electron systems has been considered by Lam and Platzman. ${ }^{33}$ Their analysis shows that the corrections to a formula analogous to Eq. (5. 7) come from the exchange-correlation part of the energy functional. In our case, the positron-induced momentum enhancement and the deviations in the momentum distribution of an interacting versus a noninteracting electron system are known largely to cancel each other. ${ }^{10}$ Consequently, one would expect that the error in Eq. (5.7), caused by the $\psi_{i}$ 's not being the true eigenstates, is insignificant in the double-integrated-long-slit angular distribution (5.8). The calculations are carried through only in the case of aluminum, where the contribution of the core electrons to the angular-correlation curve is small.

For comparison we also calculated the angularcorrelation curve by using the mixed-density approximation suggested by Arponen et al. ${ }^{13}$ In the mixed-density approximation, which is based on the differential Thomas-Fermi theory, the momentum density of the annihilating electron-positron pair is expressed as

$$
\begin{aligned}
\rho(\overrightarrow{\mathrm{p}}) \propto & \iint d \overrightarrow{\mathrm{r}} d \overrightarrow{\mathrm{r}}^{\prime} e^{i \overrightarrow{\mathrm{p}} \cdot\left(\overrightarrow{\mathrm{r}}-\overrightarrow{\mathrm{r}}^{\prime}\right)} \psi_{+}(\overrightarrow{\mathrm{r}}) \psi_{+}\left(\overrightarrow{\mathrm{r}}^{\prime}\right) \\
& \times g\left(k_{F}(\overrightarrow{\mathrm{R}})\left|\overrightarrow{\mathrm{r}}-\overrightarrow{\mathrm{r}}^{\prime}\right|\right) \Gamma(n(\overrightarrow{\mathrm{R}})),
\end{aligned}
$$

where $\overrightarrow{\mathbf{R}}=\frac{1}{2}\left(\overrightarrow{\mathbf{r}}+\overrightarrow{\mathbf{r}}^{\prime}\right)$ and the function $g$ is

$$
g(x)=\frac{3}{x^{3}}(\sin x-x \cos x) .
$$

In Eq. (5.9) the momentum distribution is computed directly from electron density $n(\vec{r})$. In Fig. 6 we have plotted the negative derivates of the angular-correlation curves normalized to equal areas. The experimental points are those of Kusmiss and Stewart, ${ }^{34}$ from which the annihilation with core electrons have been subtracted. One observes a reasonably good agreement between the experimental points and both the mixed density and the single-particle approximations. This fact gives further support for the use of Eq. (5.9) in calculating the momentum distributions of nonhomogeneous systems. Actually, the results are equally good even when the TF electron density is used. ${ }^{13}$ A common feature in both approximations is that they seem to overestimate the portion of annihilation at large momentum values. This is perhaps 


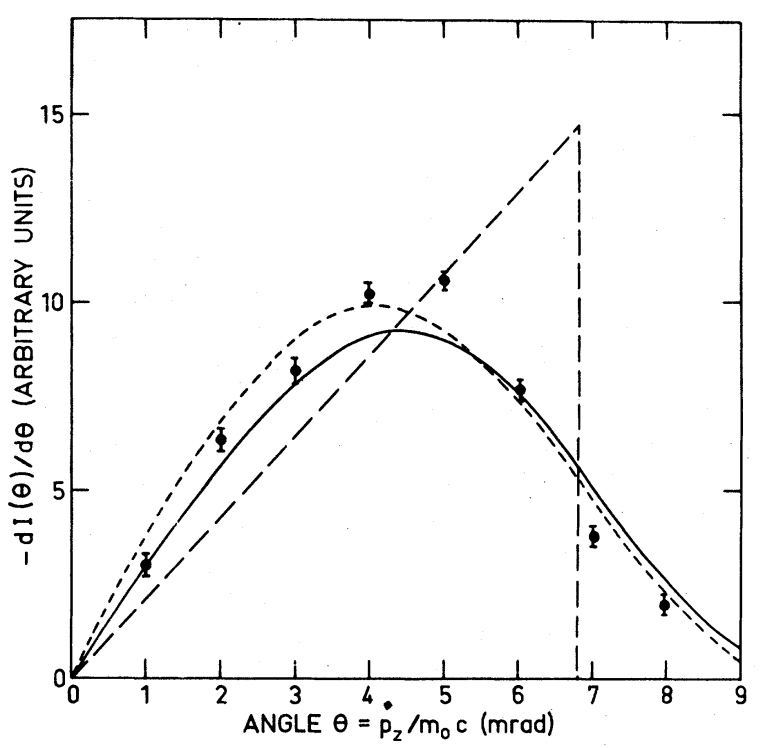

FIG. 6. Derivatives for the angular-correlation curves of positrons trapped at a vacancy of aluminum. The solid line is the curve calculated from the single-particle wave functions [Eq. (5.7)], the dotted line from the mixed density approximation [Eq. (5.9)]. The experimental points are from Kusmiss and Stewart (Ref. 34). The dashed line corresponds to the free-electron parabola. The derivatives have been calculated from the angularcorrelation curves normalized to equal areas.

not very serious, since it is not quantitatively understood how to extract the core-annihilation part from the angular-correlation curve, and, consequently, the experimental results, especially at large angles, may be rather dubious.

Finally, it can be mentioned that a strictly local approximation as suggested by Brandt ${ }^{35}$ does not seem to be very useful, when the density variations are as rapid and large as in the case of a vacancy. ${ }^{13}$

\section{CONCLUSIONS}

The Kohn-Sham approach applied to a small spherical void in the background charge suggests that the electron density inside a vacancy in metal is rather high, about one-fifth of the bulk value. The result was found to be quite insensitive to the smoothening of the background edge at the WignerSeitz radius. As a result of comparison of various methods, we can conclude that within the chosen vacancy model the statistical TF method describes fairly well the average electron density in all simple metals. Improvements to the TF method, a local exchange term and a gradient correction to the kinetic energy, do not, in the average, bring the results closer to the KS electron density. The most notable difference between the $\mathrm{KS}$ and statistical methods is that the KS electron density undergoes the correct Friedel oscillations, which are lacking in the statistical theories. This point is of great importance, e.g., in evaluating the interactions between vacancies and other imperfections in metals.

The vacancy-formation energies are very sensitive to the specific lattice model used together with the jellium model electron density. The results are reasonable for the alkali metals, where the effects of lattice relaxation and proper screening of the ionic charges do not seem to play a crucial role. The validity of our approach is questionable in metals with high $Z$ or small $r_{s}$, where the results are poorest.

The lifetimes of positrons trapped at vacancies calculated from the $\mathrm{KS}$ and TF electron densities are in good agreement with each other and also to a great extent with experimental results. The main difference is that the TF method leads to a higher electrostatic potential and thus tends to overestimate the binding energy of the trapped positron when compared to the results of the KS method. The angular-correlation curves evaluated from the KS single-particle wave functions on one hand and from the $\mathrm{KS}$ or TF electron densities on the other do not differ appreciably from each other and are in agreement with experiment. We can conclude that in calculating the annihilation characteristics of positrons in imperfections of simple metals the statistical TF theory together with the mixed-density approximation are as good as the more refined KS method and computationally much simpler and more practical.

\section{ACKNOWLEDGMENT}

We would like to thank Dr. Mike Finnis for discussions and Dr. P. de Chatel and Dr. G. Robinson for pointing out an error in the manuscript.

\section{APPENDIX: NUMERICAL SOLUTION OF THE KS ELECTRON DENSITIES}

The TF potential was chosen as the initial potential $V_{\text {eff }}^{(0)}$ in the self-consistency problem of Eqs. (2.8)-(2.11). By solving Eqs. (2.8)-(2.11) numerically we get a new potential $V_{\text {eff }}^{(1)}$, which again can be inserted into Eq. (2.8). This procedure has to be continued until the potential $V_{\text {eff }}^{(i)}$ does not change in successive iterations. However, it was found that by solving the electrostatic potential in each iteration cycle from the equation

$$
\phi^{(i)}(\overrightarrow{\mathbf{r}})=\int d \overrightarrow{\mathbf{r}}^{\prime} \frac{n_{+}\left(\overrightarrow{\mathbf{r}}^{\prime}\right)-n^{(i-1)}\left(\overrightarrow{\mathbf{r}}^{\prime}\right)}{\left|\overrightarrow{\mathbf{r}}-\overrightarrow{\mathbf{r}}^{\prime}\right|}
$$

the procedure always diverged. This led us to try the method which was found to be successful in solving the TF equation. ${ }^{13}$ The Poisson equation is written in the form

$$
\nabla^{2} \phi-k^{2} \phi=-4 \pi\left(n_{+}-n\right)-k^{2} \phi,
$$


which has a recursive solution

$$
\begin{aligned}
\phi^{(i)}(\overrightarrow{\mathrm{r}})= & \int d \overrightarrow{\mathbf{r}}^{\prime} \frac{e^{-k\left|\overrightarrow{\mathbf{r}}-\overrightarrow{\mathbf{r}}^{\prime}\right|}}{4 \pi\left|\overrightarrow{\mathbf{r}}-\overrightarrow{\mathbf{r}}^{\prime}\right|} \\
& \times\left\{-4 \pi\left[n_{+}\left(\overrightarrow{\mathrm{r}}^{\prime}\right)-n^{(i-1)}\left(\overrightarrow{\mathrm{r}}^{\prime}\right)\right\}-k^{2} \phi^{(i-1)}\left(\overrightarrow{\mathrm{r}}^{\prime}\right)\right\} .
\end{aligned}
$$

This equation leads to a sufficient convergence after about 10 iterations when starting from the TF potential. For the arbitrary constant $k$ a convenient numerical value is afforded by the TF screening constant $\left(4 k_{F} / \pi\right)^{1 / 2}$.

In metals with low electron density $(\mathrm{Na}, \mathrm{K}$, and $\mathrm{Cs})$, the exchange-correlation part of the potential is dominating. For these metals the convergence became faster when the exchange-correlation po- tential was calculated from the electron density, which was renormalized after each iteration to fulfill the charge neutrality condition.

The total number of one-particle wave functions used in calculating the displaced charge was about 200. Each wave function $u_{k, l}$ was calculated up to the radius of about $3.3 R_{\mathrm{wS}}$, where it was matched with its asymptotic sinewave of unit amplitude

$$
u_{k, l}(r) \rightarrow \frac{1}{k} \sin \left(k r-\frac{l \pi}{2}-\delta_{l}(k)\right) .
$$

The numerical accuracy of the procedure was tested by calculating the electron density for a zero potential $V_{\text {eff }} \equiv 0$. The electron density found by this method was uniform to an accuracy of $0.1 \%$.
${ }^{1}$ P. A. Johnson, J. Phys. F 3, 295 (1973).

${ }^{2}$ T. E. Faber, An Introduction to Liquid State Physics (Cambridge U. P. , Cambridge, 1972).

${ }^{3}$ P. S. Ho and R. Benedek, IBM J. Res. Dev. $\underline{18}, 386$ (1974).

${ }^{4}$ N. H. March, J. Phys. F 3, 233 (1973).

${ }^{5}$ M. J. Stott, S. Baranovsky, and N. H. March, Proc. R. Soc. A 316, 201 (1970):

${ }^{6}$ J. L. Beeby, Proc. R. Soc. A 302, 113 (1967).

${ }^{7}$ R. Nieminen, M. Manninen, P. Hautojärvi, and J. Arponen, Solid State Commun. 16, 831 (1975).

${ }^{8}$ W. Kohn and L. J. Sham, Phys. Rev. 140, A1133 (1965).

${ }^{9}$ N. D. Lang and W. Kohn, Phys. Rev. B 1, 4555 (1970).

${ }^{10}$ R. N. West, Adv. Phys. 22, 264 (1973); I. Ya. Dekhtyar, Phys. Rep. 9c, 243 (1974).

${ }^{11} \mathrm{P}$. Hohenberg and W. Kohn, Phys. Rev. 136, B864 (1964).

${ }^{12}$ L. Hedin and B. I. Lundqvist, J. Phys. C $\underline{4}, 2064$ (1971).

${ }^{13}$ J. Arponen, P. Hautojärvi, R. Nieminen, and E. Pajanne, J. Phys. F 3 , 2092 (1973).

${ }^{14} \mathrm{P}$. Gombás, Die statistische Theorie des Atoms und ihre Anwendungen (Springer-Verlag, Vienna, 1949).

${ }^{15}$ D. A. Kirzhnits, Zh. Eksp. Teor. Fiz. 32, 115 (1957) [Sov. Phys. -JETP 5, 64 (1957)].

${ }^{16}$ C. F. v. Weizsäcker, Z. Phys. 96, 431 (1935).

${ }^{17}$ W. Jones and W. H. Young, J. Phys. C 4, 1322 (1971).

${ }^{18}$ D. J. W. Geldart, M. Rasolt, and R. Taylor, Solid State Commun. 10, 279 (1972).
${ }^{19}$ G. Niklasson, A. Sjölander, and K. S. Singwi, Phys. Rev. 11, 113 (1975).

${ }^{20} \mathrm{P}$. Minchin, A. Meyer, and W. H. Young, J. Phys. F 4, 2117 (1974).

${ }^{21} \bar{P}$. S. Ho, Phys. Rev. B $\underline{3}, 4035$ (1971).

${ }^{22}$ S. P. Singhal, Phys. Rev. B $\underline{8}, 3641$ (1973).

${ }^{23}$ N. W. Asheroft, Phys. Lett. 23A, 48 (1966).

${ }^{24}$ W. M. Shuy and G. D. Gaspari, Phys. Rev. 170, 687 (1968).

${ }^{25} \mathrm{M}$. W. Finnis (unpublished).

${ }^{26}$ R. Chang and L. M. Falicov, J. Phys. Chem. Solids 32,465 (1971).

${ }^{27} \bar{C}$. H. Hodges and M. J. Stott, Phys. Rev. B $\underline{7}, 73$ (1973).

${ }^{28} \mathrm{P}$. Bhattacharyya and K. S. Singwi, Phys。 Lett. $\underline{41 \mathrm{~A}}$, 457 (1972).

${ }^{29}$ W. Brandt and J. Reinheimer, Phys. Lett. 35A, 109 (1971).

${ }^{30}$ R. N. West, Solid State Commun. 9, 1417 (1971).

${ }^{31}$ I. K. MacKenzie, T. W. Craig, and B. T. A. McKee, Phys. Lett. 36A, 227 (1971).

${ }^{32}$ W. Brandt, Appl. Phys. 5 , 1 (1974).

${ }^{33}$ L. Lam and P. M. Platzman, Phys. Rev. B 9, 5122 (1974).

${ }^{34}$ J. H. Kusmiss and A. T. Stewart, Adv. Phys. 16, 471 (1967).

${ }^{35}$ W. Brandt, in Positron Annihilation, edited by A. T. Stewart and L. O. Roellig (Academic, New York, 1967), p. 176. 